\title{
Sobrevivência específica de pacientes com câncer de pulmão tratados no sistema público de saúde no Brasil e uma aplicação da Tábua Associada de Decremento Único
}

\author{
Carlos Philipe Barbosa Polato* \\ Pamila Siviero** \\ Carla Jorge Machado ${ }^{* * *}$ \\ Gisele Macedo da Silva Bonfante ${ }^{* * * *}$ \\ Eli lola Gurgel Andrade ${ }^{* * * * *}$ \\ Francisco de Assis Acurcio \\ Mariângela Leal Cherchiglia ${ }^{+* x+x+x}$
}

\section{Introdução}

Segundo o National Cancer Institute (2012) dos Estados Unidos, algumas medidas de sobrevivência por câncer são importantes, entre as quais duas se destacam. A primeira é a sobrevivência líquida específica por câncer (netcancer-specific survival), que é uma estatística importante para formulação de políticas públicas destinadas à redução da mortalidade pelo câncer: trata-se da probabilidade de sobreviver ao câncer na ausência de outras causas de morte. Essa medida não é influenciada por mudanças na mortalidade por outras causas e, portanto, fornece um indicador útil para rastrear a sobrevivência ao longo do tempo e possibilita comparações entre diferentes países. A segunda é a probabilidade bruta de morte (crude probability of death) (medida prognóstico do paciente), que corresponde à probabilidade de morrer de câncer na presença de outras causas de morte. Trata-se de uma medida mais indicada para avaliar o impacto do diagnóstico de câncer em um nível individual, uma vez que a mortalidade por outras causas desempenham papel fundamental no padrão de mortalidade de uma população. Ela mensura os padrões de mortalidade experimentados

\footnotetext{
*Hospital Municipal Odilon Behrens, Belo Horizonte-MG, Brasil (philipepolato@hotmail.com).

** Universidade Federal de Alfenas, Alfenas-MG, Brasil (pclsiviero@gmail.com).

${ }^{* \star *}$ Universidade Federal de Minas Gerais - UFMG, Belo Horizonte-MG, Brasil (carlajm@ufmg.br).

**** Exército Brasileiro - EB, Belo Horizonte-MG, Brasil (gimacedosilva@hotmail.com).

***** Universidade Federal de Minas Gerais - UFMG, Belo Horizonte-MG, Brasil (iola@medicina.ufmg.br).

****** Universidade Federal de Minas Gerais - UFMG, Belo Horizonte-MG, Brasil (acurcio@ufmg.br).

******* Universidade Federal de Minas Gerais - UFMG, Belo Horizonte-MG, Brasil (cherchml@medicina.ufmg.br).
} 
em uma coorte de pacientes com câncer, na qual outras causas de morte estão atuando simultaneamente.

No Brasil, os estudos sobre sobrevivência específica por câncer são baseados apenas na segunda medida (chamada aqui de probabilidade bruta). Nesta situação, indivíduos que morrem por outras causas são classificados como censuras e uma tabela de vida é obtida, ou, ainda, uma estimativa com base no estimador de Kaplan-Meier (CINTRA et al., 2008; MIGOWSKI; SILVA, 2010).

Há outra forma muito utilizada por demógrafos e atuários, mas ainda pouco empregada na saúde pública, que converge com a ideia de sobrevivência líquida específica por câncer, cujo ponto de partida é a obtenção de uma tabela associada de decremento único (BOWERS et al., 1997; BORGES; BELTRÃO, 2010). Especifica-se, neste trabalho, como produzir estimativas de sobrevivência com base na geração de uma tabela associada de decremento único, além de se obter a diferença entre estimativas de óbito e de sobrevivência acumulada alcançadas com base nas probabilidades brutas (por meio das tábuas de múltiplos decrementos) e líquidas (provenientes da tábua associada de decremento único). Para este exercício, foi escolhido o câncer de pulmão, dado que é o segundo câncer mais incidente em homens e o quinto em mulheres no Brasil, excetuando-se o câncer de pele não melanoma (INCA, 2011). Nos Estados Unidos, o câncer de pulmão é a primeira causa de morte por câncer em homens e mulheres (SIEGEL, 2012).

Assim, neste trabalho, realizou-se análise de sobrevida por câncer de pulmão inferior a um ano e superior a cinco anos dos respectivos pacientes.

\section{Dados e métodos}

Os dados referem-se a pacientes submetidos à radioterapia e/ou quimioterapia com ou sem cirurgia associada para tratamento do câncer de pulmão no SUS, entre 01 de janeiro de 2002 e 31 de dezembro de 2003.

As informações são disponibilizadas no subsistema Autorização para Procedimentos de Alto Custo/Complexidade - Apac do Sistema de Informações Ambulatoriais - SIA/SUS, com seleção dos procedimentos de radioterapia e/ou quimioterapia. A aplicação da técnica de pareamento determinístico-probabilístico permitiu a geração de um cadastro único de pacientes que realizaram tratamento radioterápico e/ou quimioterápico no SUS entre 2000 e 2006.

Para cada paciente foram reunidos todos os dados sobre tratamento oncológico ambulatorial (radioterapia, quimioterapia, combinado) realizado no SUS.

Os dados relativos à ocorrência de óbitos foram confirmados por meio de pareamento determinístico-probabilístico entre a Base Onco e o Sistema de Informações sobre Mortalidade SIM, disponibilizado para 2003 a 2008.

Para a coorte de 11.167 indivíduos, obtiveram-se as probabilidades brutas de sobrevivência mensais entre dois meses exatos até o tempo exato de 60 meses (cinco anos). 
Após este período de tempo, optou-se por não calcular novas estimativas em decorrência de várias saídas da coorte por remissão do paciente com câncer de pulmão (ou seja, saídas diferentes de óbito).

O fundamento básico da teoria de múltiplos decrementos consiste em considerar a distribuição de duas variáveis aleatórias relacionadas a um único indivíduo: o tempo até o término de determinado status, $T(x)$; e a causa desse término, $J(x)$ (BORGES; BELTRÃO, 2010). No caso específico de estudos de sobrevivência, $T(x)$ indica o tempo até a morte, ao passo que $J(x)$ representa as $m$ causas que podem levar o indivíduo à morte.

Assim, em um ambiente multidecremental, no qual diversas causas concorrem para levar o indivíduo ao óbito, torna-se possível definir a probabilidade de morte por todas as causas $\left({ }_{n} q_{x}^{\tau}\right)$, que pode ser expressa por:

$$
{ }_{n} q_{x}^{\tau}=\sum_{j=1}^{m}{ }_{n} q_{x}^{j}
$$

onde ${ }_{n} q_{x}^{j}$ representa a probabilidade bruta de morte entre os meses $\mathrm{x}$ e $(\mathrm{x}+\mathrm{n})$, por determinada causa $(j)$, ocorrer em um ambiente multidecremental, ou seja, na presença de outras causas de morte atuando de forma simultânea.

Supõe-se, ainda, que um grupo de $l^{\tau}$ indivíduos está sujeito a forças determinísticas e competitivas de decrementos. Nesse sentido, é possível definir o número de óbitos esperados na presença de outras causas de decremento:

$$
{ }_{n} d_{x}^{\tau}=l_{x n}^{\tau} q_{x}^{\tau}=l_{x}^{\tau}-l_{x+1}^{\tau}
$$

onde $l_{x}^{t}$ indica o número de sobreviventes ao mês x. Tais indivíduos morrerão em algum momento futuro por meio de uma das $m$ causas de morte. Assim, define-se o número de óbitos esperados por cada uma das $m$ causas:

$$
{ }_{n} d_{x}^{j}=l_{x n}^{\tau} q_{x}^{j}=l_{x}^{j}-l_{x+n}^{j}
$$

em que $l_{x}^{j}$ representa o número de sobreviventes ao mês $x$ que morrerão pela causa j, sendo

$$
l_{x}^{\tau}=\sum_{j=1}^{m} l_{x}^{j}
$$

Na sequência, obteve-se o número de óbitos acumulados a partir da mês zero até o mês x:

$$
{ }_{x} d_{0}=\sum_{x=0}^{n}{ }_{x} d_{0}
$$

Foram obtidas, em seguida, a função acumulada de mortalidade $F(x)=\frac{{ }_{x} d_{0}}{l_{0}^{\tau}}$ e a função acumulada de sobrevivência $S(x)=1-F(x)$.

Na teoria dos múltiplos decrementos, para cada uma das $m$ causas de morte, é possível definir, ainda, um modelo que dependa exclusivamente de uma única causa de morte. Denominado de probabilidade condicional líquida de morte, este modelo indica qual seria a probabilidade de morrer por determinada causa, se apenas essa causa estivesse atuando. Assim, com base nas informações da tabela de múltiplo decremento, é possível construir 
tábuas de decremento único associadas à tábua de múltiplo decremento (BOWERS et al., 1997).

Nesse sentido, obteve-se a probabilidade condicional líquida de morte da tábua associada de decremento único por câncer de pulmão. Com ${ }_{n} a_{x}=0,5$

$$
{ }_{n} q_{x}^{[j]}=1-\left({ }_{n} p_{x}^{\tau}\right)^{\frac{{ }_{n} q_{x}^{j}}{{ }^{\tau}} q_{x}^{\tau}}
$$

onde ${ }_{n} p_{x}^{\tau}$ indica a probabilidade de um indivíduo vivo no mês $x$ sobreviver até o mês $(x+n)$; ${ }_{n} q_{x}^{j}$ refere-se à probabilidade de um indivíduo que estava vivo no mês $x$ morrer pela causa $j$ antes do mês $(x+n)$ e ${ }_{n} q_{x}^{t}$ representa a probabilidade de um indivíduo sobrevivente no mês $x$ morrer antes do mês $(x+n)$, independentemente da causa. As três funções são obtidas na tábua de múltiplo decremento.

Assim, com o objetivo de calcular a probabilidade condicional líquida de morte por câncer de pulmão, submeteu-se uma coorte hipotética de $\mathrm{l}(0)=100.000$ indivíduos que estariam com câncer de pulmão no mês zero a estas probabilidades mensais.

Obteve-se o número de óbitos esperados na ausência de outras causas.

${ }_{n} d_{x}^{\prime j}={ }_{n} L_{x} \cdot{ }_{n} m_{x}^{j} \cong_{n} L_{x} \cdot{ }_{n} m_{x}^{\prime j}$

onde ${ }_{n} L_{x}$ representa o número de pessoas-ano vividos entre os meses $x$ e $(x+n)$ e ${ }_{n} m_{x}^{j}$ corresponde à taxa de mortalidade entre os meses $x$ e $(x+n)$, sob o pressuposto de força da mortalidade constante a cada mês (BOWERS et al., 1997).

Na sequência, calculou-se o número de óbitos acumulados a partir do mês zero até o mês $x$ :

$$
{ }_{x} d_{0}^{\prime}=\sum_{x=0}^{n}{ }_{x} d_{0}
$$

Por fim, foram obtidas, as funções acumuladas de mortalidade e sobrevivência.

$$
F^{\prime}(x)=\frac{{ }_{x} d_{0}^{\prime}}{l_{0}^{\tau}} \quad \text { e } \quad S^{\prime}(x)=1-F^{\prime}(x)
$$

\section{Resultados}

O Gráfico 1 apresenta as probabilidades acumuladas de óbito por câncer de pulmão, de indivíduos diagnosticados entre 2002 e 2003, calculadas por meio da tábua de múltiplo decremento $(\mathrm{F}(\mathrm{x}))$ e da tábua associada de decremento único $\left(\mathrm{F}^{\prime}(\mathrm{x})\right)$.

A sobrevivência inferior a um ano (ou doze meses) foi de 47,6\% na presença de outras causas $(S(12)=1-F(12)=47,6 \%)$ e de $43,9 \%$ na ausência de outras causas ( $S^{\prime}(12)=1$ $\left.F^{\prime}(12)=43,9 \%\right)(7,8 \%$ menor). Aos 60 meses, os valores encontrados foram de $26,0 \%$ quando o câncer de pulmão concorria com outras causas para levar o indivíduo à morte $(S(60)=1$ - $F(60)=26,0 \%)$ e $16,2 \%$ se a única causa de morte fosse o câncer de pulmão (S’(60)=1 - 
$\left.F^{\prime}(60)=16,2 \%\right)$, o que implica 59,0\% de redução. Entende-se, então, o nível de subestimação da sobrevivência quando se utiliza apenas a função de sobrevivência dada em presença de outras causas de óbito. Ademais, quando se trata da sobrevivência superior a cinco anos (ou 60 meses), a subestimação é ainda maior, decorrente da diferença acumulada ao longo dos meses.

GRÁFICO 1

Probabilidades acumuladas de óbito por câncer de pulmão em indivíduos diagnosticados com a doença Brasil - 2002-2003

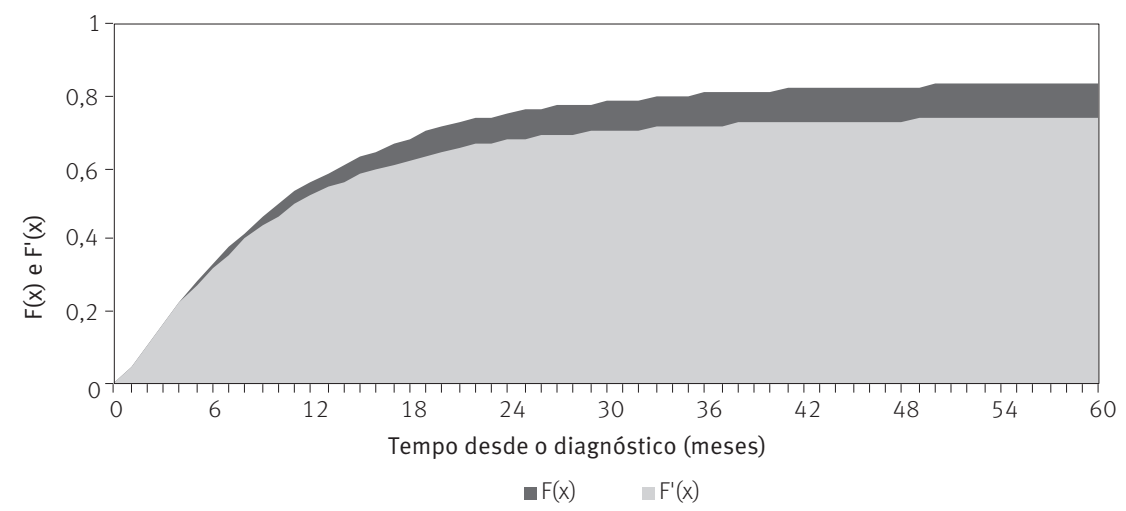

Fonte: Ministério da Saúde. Sistema de Informações Ambulatoriais - SIA/Autorização para Procedimentos de Alto Custo/Complexidade Apac; Sistema de Informações sobre Mortalidade - SIM.

\section{Conclusão}

Os resultados encontrados sugerem a subestimação da sobrevivência específica por câncer, quando se utiliza a probabilidade bruta de morte, em um ambiente multidecremental, em detrimento da sobrevivência líquida, calculada por meio da tábua associada de decremento único. Assim, levanta-se a importância de utilizar as medidas de maneira conjunta. Nesse sentido, espera-se que novos estudos na área de saúde pública possam empregar também esta nova medida, com o objetivo de ampliar a compreensão da mortalidade e da letalidade por câncer do pulmão e por outras doenças.

\section{Referências}

BORGES, G. M.; BELTRÃO, K, I. Tábua de múltiplos decrementos para a saída da atividade no funcionalismo público federal. In: 19 SINAPE - Simpósio Nacional de Probabilidade e Estatística. Anais... São Pedro, 2010.

BOWERS, N. L. et al. Actuarial mathematics. Illinois: Society of Actuaries, 1997.

CINTRA, J. R. D.; GUERRA, M. R.; BUSTAMANTE-TEIXEIRA, M. T. Sobrevida específica de pacientes com câncer de mama não-metastático submetidas à quimioterapia adjuvante. Rev. Assoc. Med. Bras., v. 54, n. 4, ago. 2008.

INSTITUTO NACIONAL DO CANCER JOSÉ ALENCAR GOMES DA SILVA. Coordenação Geral de Ações Estratégicas. Coordenação de Prevenção e Vigilância. Estimativa 2012: incidência de câncer no Brasil. Rio de Janeiro: Inca, 2011. 
MIGOWSKI, A.; SILVA, G. A. Sobrevida e fatores prognósticos de pacientes com câncer de próstata clinicamente localizado. Rev. Saúde Pública, v. 44, n. 2, abr. 2010. Disponível em: ‘http://www.scielosp. org/scielo.php?script=sci_arttext\&pid=S0034-89102010000200016\&lng=en\&nrm=iso .

NATIONAL CANCER INSTITUTE. Measures of cancer survival. 2012. Disponivel em: <http://surveillance. cancer.gov/survival/measures.html>.

SIEGEL, R.; NAISHADHAM D.; JEMAL, A. Cancer statistics, 2012. Ca Cancer J Clin, n. 62, p. 10-29, 2012.

\section{Autores}

Carlos Philipe Barbosa Polato é mestre em Saúde Pública (UFMG). Médico anestesiologista do Hospital Odilon Behrens.

Pamila Siviero é doutora em Demografia (Cedeplar/UFMG). Professora adjunta da Universidade Federal de Alfenas.

Carla Jorge Machado é Ph.D. pela Universidade Johns Hopkins/EUA. Professora associada da Universidade Federal de Minas Gerais (UFMG).

Gisele Macedo da Silva Bonfante é mestre e doutoranda em Saúde Pública (UFMG). Dentista do Exército Brasileiro.

Eli lola Gurgel Andrade é doutora em Demografia (Cedeplar/UFMG). Professora associada da Universidade Federal de Minas Gerais (UFMG).

Francisco de Assis Acurcio é doutor em Ciência Animal (área de concentração epidemiologia da UFMG). Professor titular da Universidade Federal de Minas Gerais (UFMG).

Mariângela Leal Cherchiglia é doutora em Saúde Pública (USP). Professora associada da Universidade Federal de Minas Gerais (UFMG). 